\title{
On the Implementability of Automorphism Groups
}

\author{
H. J. BORCHERS \\ Institut für Theoretische Physik der Universität Göttingen
}

Received July 9, 1969

\begin{abstract}
Let $\mathscr{A}$ be a $C^{*}$-algebra and $G$ be a locally compact group acting as strongly continuous automorphisms on $\mathscr{A}$. Let $\pi$ be a representation of $\mathscr{A}$ then we say $\pi$ is a covariant representation if there exists a strongly continuous unitary representation of the group acting on $\mathscr{H}_{\pi}$ which implements the automorphisms. We give necessary and sufficient conditions on a representation $\pi$ of $\mathscr{A}$ such that a) $\pi$ is subrepresentation of a covariant representation and $b$ ) $\pi$ is subrepresentation of a covariant representation quasi-equivalent to $\pi$.
\end{abstract}

\section{Introduction}

Almost every physical problem is connected with the action of some group, sometimes as a symmetry-group of the problem, very often as the group of time development and not seldom as the combination of both. This is also true in those cases where $C^{*}$-algebras have been used for the description of physics, as for instance quantum-field-theory and statistical mechanics. In these cases such groups appear as automorphism-groups of the $C^{*}$-algebra. Although it is widely believed that all physical information should be purely algebraic, this means, independent of the special representation [1], everyone prefers to use special representations adapted to the special situation. Common to all such representations which have been used so far is the property that the group of automorphisms which are of physical interest are implemented by a continuous unitary representation of the group in question.

At this point we interrupt the discussion in order to introduce some terminology. We denote the $C^{*}$-algebra by $\mathscr{A}$ (for the definition see J. Dixmier [2]) and by $G$ a locally compact group. We assume that we have a representation $\alpha: G \rightarrow$ Aut $\mathscr{A}$ of this group as automorphisms acting on $\mathscr{A}$ (Aut $\mathscr{A}$ denotes the automorphism-group of $\mathscr{A}$ ). Speaking about representations $\pi$ of $\mathscr{A}$ we mean always representations which are not degenerated.

I.1. Definitions. A representation $\pi$ of $\mathscr{A}$ is called

a) covariant if there exists a strongly continuous unitary representation $\varrho: G \rightarrow \mathscr{B}\left(\mathscr{H}_{\pi}\right)$ (all bounded operators on $\mathscr{H}_{\pi}$ ) such that $\pi\left(\alpha_{g} x\right)$ $=\varrho(g) \pi(x) \varrho^{-1}(g)$ for all $x \in \mathscr{A}$; 
b) quasicovariant if $\pi$ is quasi equivalent to a covariant representation $\pi^{\prime}$;

c) covariant extendible if $\pi$ is unitary equivalent to a subrepresentation $\pi_{1}$ of a covariant representation $\pi_{2}$.

We will denote by $\alpha_{g}^{\prime}$ the transposed mapping of $\alpha_{g}$ defined on the dual space of $\mathscr{A}$ by the relation $\left(\alpha_{g}^{\prime} \omega\right)(x)=\omega\left(\alpha_{g} x\right)$.

I.2. Definitions. We say the automorphisms $\alpha_{g}, g \in G$ act

a) norm continuous if for every $\varepsilon>0$ exists a neighbourhood $U \subset G$ of the identity such that $\left\|\alpha_{g} x-x\right\| \leqq \varepsilon\|x\|$ for $g \in U$;

b) strongly continuous if for every $x \in \mathscr{A}$ and every $\varepsilon>0$ exists a neighbourhood $U \subset G$ of the identity such that $\left\|\alpha_{g} x-x\right\|<\varepsilon$ for $g \in U$.

Since only covariant representations seem to be of use for physics it is natural to ask for conditions under which a representation will be a covariant one. This question has been solved by R. Kadison and J. Ringrose [3] in the case where the group acts norm continuous, using the result of S. Sakai on derivations [4]. However, it is also known that norm continuous groups of automorphisms are not useful in physics because of too much analyticity [5]. The next class to ask for would be automorphism-groups acting strongly continuous on the algebra. Indeed, from examples in field theory and in statistical mechanics it is known that this situation does occur in physics.

Most investigations of covariant representations are based on invariant states where the covariance of the representation is guaranteed by the invariance of the state (see e.g. Doplicher, Kadison, Kastler, and Robinson [6] where one finds also further references). A completely different piece of information has been found by Doplicher, Kastler, and Robinson [7] namely that covariant representations are in one to one correspondence with representations of a related algebra which they called covariance algebra. But also here no attempt has been made to give conditions on the representations which guarantee that this representation is a covariant one. The first real attempt of proving the covariance of a given representation has been made in connection with abelian groups and the spectrum condition by G. F. Dell'Antonio [8]. Recently H. J. Borchers [9] gave necessary and sufficient conditions for a representation being covariant with a group-representation fulfilling the spectrum condition. This case, however, has been a favourable one since the spectrum condition implies, according to Borchers [10], that the group representation, if it exists, can be chosen in the weak closure of the representation.

If one tries to give conditions for the covariance of a representation in the general situation it becomes clear that one has to cope with cohomology and with multiplicity problems. In order that we do not have 
to deal with these problems we will restrict ourselves to the case of finding conditions under which a representation is covariant extendible respectively under which it is a quasi covariant representation. These problems can be solved and have simple answers.

In the following it is always assumed that $G$ acts as a strongly continuous group of automorphisms.

\section{States which are Continuous under the Action of the Groups}

We will denote by $E(\mathscr{A})$ the set of states on $\mathscr{A}$ and by $P(\mathscr{A})$ the set of pure states of $\mathscr{A}$.

II.1. Definition. We say $\alpha_{g}^{\prime}$ acts strongly continuous on a state $\omega \in E$ if $\left\|\alpha_{g}^{\prime} \omega-\omega\right\| \rightarrow 0$ for $g \rightarrow 1$ and denote by $E_{c}$ the set of states such that $\alpha_{g}^{\prime}$ acts strongly continuous on them.

The importance of this set follows from

II.2. Lemma. Let $\pi$ be a covariant representation then every vectorstate of $\pi$ belongs to $E_{c}$.

Proof. Let $\varrho: G \rightarrow \mathscr{B}\left(\mathscr{H}_{\pi}\right)$ be the strongly continuous unitary representation of $G$ implementing the automorphisms $\alpha_{g}$ and $\xi \in \mathscr{H}_{\pi}$ the $\alpha_{g}^{\prime} \omega_{\xi}(x)=\omega_{\xi}\left(\alpha_{g} x\right)=\left(\varrho^{-1}(g) \xi, \pi(x) \varrho^{-1}(g) \xi\right)$. From this follows $\left|\alpha_{g}^{\prime} \omega_{\xi}(x)-\omega_{\xi}(x)\right|=\left|\left(\left(\varrho^{-1}(g)-1\right) \xi, \pi(x) \varrho^{-1}(g) \xi\right)+\left(\xi, \pi(x)\left(\varrho^{-1}(g)-1\right) \xi\right)\right|$ $\leqq 2\|x\|\left\|\left(\varrho^{-1}(g)-1\right) \xi\right\| \rightarrow 0$ for $g \rightarrow 1$ since $\varrho$ is a strongly continuous representation. But this implies $\left\|\alpha_{g}^{\prime} \omega-\omega\right\| \rightarrow 0$ for $g \rightarrow 1$.

This Lemma tells us that states giving rise to covariant extendible representations must lie in $E_{c}$. Thir makes it worthwhile to study $E_{c}$ more closely. Its properties are collected in

II.3. Theorem. $E_{c}$ has the following properties:

1. $E_{c}$ is convex.

2. $E_{c}$ is norm closed.

3. $E_{c}$ is invariant under $\alpha_{g}^{\prime}$.

4. $\omega \in E_{c}$ and $x \in \mathscr{A}$ such that $\omega\left(x^{*} x\right)=1$ then $\omega_{x} \in E_{c}$ where $\omega_{x}(y)$ $=\omega\left(x^{*} y x\right)$.

5. $\omega \in E_{c}$ and $\omega=\lambda \omega_{1}+(1-\lambda) \omega_{2}$ with $0<\lambda<1$ and $\omega_{1}, \omega_{2} \in E$ then follows $\omega_{1}, \omega_{2} \in E_{c}$.

6. $\omega \in E$ then exists a unique decomposition $\omega=\lambda \omega_{1}+(1-\lambda) \omega_{2}$ with $0 \leqq \lambda \leqq 1 . \omega_{1} \in E_{c}$ and $\omega_{2} \in E$ such that $\omega_{2}$ does not majorise any state belonging to $E_{c}$.

7. There exists a family $E_{c}^{\beta} \subset E_{c}$, indexed by a semiordered set, such that

a) $E_{c}^{\beta} \subset E_{c}^{\gamma}$ for $\beta<\gamma$,

$\beta) E_{c}^{\beta}$ is convex and weakly closed and invariant under $\alpha_{g}^{\prime}$,

r) $\bigcup_{\beta} E_{c}^{\beta}=E_{c}$.

The rest of this section is devoted to prove this theorem. 
Proof. 1. Let $\omega_{1}, \omega_{2} \in E_{c}$ and $0 \leqq \lambda \leqq 1$ then $\| \alpha_{g}^{\prime}\left(\lambda \omega_{1}+(1-\lambda) \omega_{2}\right)$ $-\left(\lambda \omega_{1}+(1-\lambda) \omega_{2}\right)\|=\| \lambda\left(\alpha_{g}^{\prime} \omega_{1}-\omega_{1}\right)+(1-\lambda)\left(\alpha_{g}^{\prime} \omega_{2}-\omega_{2}\right) \|$ $\leqq \lambda\left\|\alpha_{g}^{\prime} \omega_{1}-\omega_{1}\right\|+(1-\lambda)\left\|\alpha_{g}^{\prime} \omega_{2}-\omega_{2}\right\| \rightarrow 0$ for $g \rightarrow 1$.

2 . For proving the second statement we remark first that the transposed mapping of an automorphism is a norm preserving map of the dual space $\mathscr{A}^{*}$ of $\mathscr{A}$. Let now $\omega$ be a limit point in norm of $E_{c}$ then exists a sequence $\omega_{n} \in E_{c}$ such that $\left\|\omega_{n}-\omega\right\| \rightarrow 0$ for $n \rightarrow \infty$. Hence also $\left\|\alpha_{g}^{\prime} \omega_{n}-\alpha_{g}^{\prime} \omega\right\| \rightarrow 0$. Let now $\varepsilon>0$ then exists $n$ such that $\left\|\omega_{n}-\omega\right\| \leqq \varepsilon / 3$ and $U$ such that $\left\|\alpha_{g}^{\prime} \omega_{n}<\omega_{n}\right\| \leqq \varepsilon / 3$ for $g \in U$. Hence $\left\|\alpha_{g}^{\prime} \omega-\omega\right\|$ $\leqq\left\|\alpha_{g}^{\prime}\left(\omega-\omega_{n}\right)\right\|+\left\|\omega_{n}-\omega_{n}\right\|+\left\|\omega_{n}-\omega\right\| \leqq \varepsilon$ for $g \in U$. This implies $\alpha_{g}^{\prime}$ acts strongly continuous on $\omega$ and hence $\omega \in E_{c}$.

3. This follows immediately from the group property $\left\|\alpha_{g}^{\prime} \alpha_{h}^{\prime} \omega-\alpha_{h}^{\prime} \omega\right\|$ $=\left\|\alpha_{h^{-1} g_{h}}^{\prime} \omega-\omega\right\| \rightarrow 0$ for $g \rightarrow 0$.

4. This follows from the relation

$$
\begin{aligned}
\alpha_{g}^{\prime} \omega_{x}(y)-\omega_{x}(y)= & \omega\left(x^{*} \alpha_{g}(y) x\right)-\omega\left(x^{\prime \prime} y x\right) \\
= & \left(\omega\left(x^{\prime \prime} \alpha_{g}(y) x\right)-\omega\left(\alpha_{g^{-1}}\left(x^{\prime \prime}\right) y \alpha_{g^{-1}}(x)\right)\right) \\
& +\left(\omega\left(\alpha_{g^{-1}}\left(x^{*}\right) y \alpha_{g^{-1}}(x)\right)-\omega\left(\alpha_{g^{-1}}\left(x^{*}\right) y x\right)\right) \\
& +\left(\omega\left(\alpha_{g}^{-1}\left(x^{*}\right) y x\right)-\omega\left(x^{*} y x\right)\right) .
\end{aligned}
$$

This implies $\left\|\alpha_{g}^{\prime} \omega_{x}-\omega_{x}\right\| \leqq\left\|\alpha_{g}^{\prime} \omega-\omega\right\|\|x\|^{2}+2\left\|\alpha_{g}^{-1} x-x\right\| \cdot\|x\| \rightarrow 0$ for $g \rightarrow 1$.

5. From 4 . and 2 . follows that with any state $\omega \in E_{c}$ also every vectorstate belonging to $\pi_{\omega}$ is an element of $E_{c}$ ([2] 2.4.8). Now every state majorised by $\omega$ is a vectorstate ([2], 2.5.1). This implies 5 .

6 . We have seen that with any state $\omega \in E_{c}$ also every vectorstate belonging to $\pi_{\omega}$ is an element of $E_{c}$. Since $E_{c}$ is convex and norm closed we see that all vectorstates of direct sums of respresentations, whose vectorstates are elements of $E_{c}$, belong also to $E_{c}$. We will collect these results in a proposition. To this end we give first a

II.4. Definition. We say a representation $\pi$ is affiliated to $E_{c}$ if every vectorstate belonging to $\pi$ is an element of $E_{c}$. We write $\pi \eta E_{c}$.

II.5. Proposition. 1. Let $\pi \eta E_{c}$ and $\pi_{1}$ be a subrepresentation of $\pi$ then $\pi_{1} \eta E_{c}$.

2. Assume $\pi_{i} \eta E_{c}$, $i \in I$ then $\sum_{i \in I} \oplus \pi_{i} \eta E_{c}$.

3. Let $\pi \eta E_{c}$ and $\pi_{1}$ quasiequivalent to $\pi$ then $\pi \eta E_{c}$.

Proof. The first statement follows immediately from the definition of affiliated representations. The second statement follows from the remarks given just before the definition II.4. It remains the third statement. Since $\pi$ and $\pi_{1}$ are quasiequivalent there exists a representation $\pi_{2}$ quasi equivalent to $\pi$ such that $\pi$ and $\pi_{1}$ can be identified with sub- 
representations of $\pi_{2}$ ([11], I, $\S 4$ Corollaire). Taking into account the result of statement 1 . we need only to prove that $\pi_{2}$ is affiliated to $E_{c}$. Now, according to ([2], 5.3.1) we can choose for $\pi_{2}$ a multiple of $\pi$. Hence $\pi_{2} \eta E_{c}$ by the second statement.

We are now turning back to the proof of the theorem. Let us denote by $\pi=\sum_{\omega \in E_{c}} \oplus \pi_{\omega}$ which is by Proposition II.5. affiliated to $E_{c}$. If we denote by $\pi_{u}$ the universal representation then exists a unique central projection $P$ of the weak closure of $\pi_{u}$ such that $\pi$ is quasi equivalent to $P \pi_{u}$ and therefore $P \pi_{u} \eta E_{c}$ by Proposition II.5. On the other hand if $\omega$ is a vectorstate of $(1-P) \pi_{u}$ then $\alpha_{g}^{\prime}$ does not act strongly continuous on $\omega$ by construction of $P$. By definition of the universal representation $\pi_{u}$ every state $\omega$ is a vector state $\omega_{\xi}$. Now $\lambda \omega_{1}=\omega_{p \xi},(1-\lambda) \omega_{2}=\omega_{(1-p) \xi}$ with $\lambda=\|P \xi\|$ gives the decomposition of $\omega_{\xi}$ such that $\omega_{1} \in E_{c}$ and $\omega_{2}$ does not majorise any state belonging to $E_{c}$. The last statement follows from the fact that every state majorised by $\omega_{2}$ is a vectorstate of $(1-P) \pi_{u}$.

For proving the last part of Theorem II.3 we remark first that for any $\omega \in E_{c}$ the expression $\left\|\alpha_{g}^{\prime} \omega-\omega\right\|$ defines a continuous nonnegative function on $G$ which vanishes at the identity. We call $I_{1}(G)$ the maximal ideal of bounded continuous functions vanishing at the identity, $I_{1}^{+}(G)$ is a semiordered set.

II.6. Definition. For every function $\beta \in I_{1}^{+}(G)$ we define $E_{c}^{\beta}$ as the set of $\omega \in E_{c}$ such that $\left\|\alpha_{g}^{\prime} \omega-\omega\right\| \leqq \beta(g)$.

It follows from the definition of $E_{c}^{\beta}$ that $E_{c}^{\beta} \subset E_{c}^{\gamma}$ for $\beta<\gamma$. Furthermore, every $\omega$ belongs to all $E_{c}^{\beta}$ with $\beta(g) \geqq\left\|\alpha_{g}^{\prime} \omega-\omega\right\|$ hence $\bigcup_{\beta} E_{c}^{\beta}=E_{c}$. Since the set of functions which are smaller then a given one is a convex set follows that $E_{c}^{\beta}$ is a convex set. Let now $\omega_{\alpha}$ be a weakly convergent net in $E_{c}^{\beta}$ then we have for every $x \in \mathscr{A}$ the relation $\left|\omega_{\alpha}\left(\alpha_{g} x-x\right)\right| \leqq\|x\| \cdot \beta(g)$ hence this relation holds also for the limit point $\omega$, this means $\omega \in E_{c}^{\beta}$.

II.7. Remarks. 1. We want to emphasize that the extremal points of $E_{c}^{\beta}$ are not pure states in general. This can easily be seen from the example of continuous functions on $G$ vanishing at infinity having the translations as a strongly continuous group of automorphisms.

2. From the fact that $\alpha_{g}$ acts strongly continuous on $\mathscr{A}$ follows that $E_{c}$ is not empty. For $f \in \mathscr{L}_{1}(G)$ with $f \geqq 0$ and $\int f(g) d g=1$ defines $x \rightarrow x(f)=\int f(g) \alpha_{g} x d g$ a linear order preserving map from $\mathscr{A} \rightarrow \mathscr{A}$. This implies for any $\omega \in E$ we have a state $\omega_{f}(x)=\omega(x(f))$. Now

$$
\begin{array}{r}
\left|\left(\alpha_{g}^{\prime} \omega_{f}-\omega\right)(x)\right|=\left|\omega\left(x\left(f_{g}\right)-x(f)\right)\right|\left|\omega\left(x\left(f_{g}-f\right)\right)\right| \leqq\|x\|\left\|f_{g}-f\right\|_{\mathscr{L}_{1} \rightarrow 0} \\
\text { for } \quad g \rightarrow 1
\end{array}
$$

This implies $\omega_{f} \in E_{c}\left(f_{g}(h)=f\left(g^{-1} h\right)\right)$. 


\section{Construction of Covariant Representations}

In this section we want to prove that every state belonging to $E_{c}$ is vectorstate of a covariant representation. The main result of this section will be

III.1. Theorem. The following statements are equivalent

1. $\pi$ is covariant extendible.

2. $\pi \eta E_{c}$ (see II.4. for the definition of affiliation).

3. $\pi$ is the direct sum of cyclic representations such that the states $\omega_{\xi_{i}},\left\{\xi_{i}\right\}_{i \in I}$ the cyclic vectors, belong to $E_{c}$.

Proof of the first part: $1 . \Rightarrow 2$. follows from Lemma II.2.

2 . $\Rightarrow 3$. Since every representation is direct sum of cyclic representations $([2], 2.2 .7)$.

3. $\Rightarrow 2$. $\omega_{\xi_{i}}$ belongs to $E_{c}$. Hence $\pi_{\omega_{\xi_{1}}} \eta E_{c}$ by Theorem II.3, 4 and 2 . Hence $\pi=\sum_{i \in I}^{\xi_{i}} \oplus \pi_{\omega_{\xi_{i}}} \eta E_{c}$ by Proposition II.5.

For proving the implication $2 . \Rightarrow 1$. we will use the covariance algebras introduced by Doplicher, Kastler, and Robinson [7]. For the covenience of the reader we will collect the main definitions and facts about these algebras.

One defines $\mathscr{A}_{1}^{G}$ as the set of functions $F: G \rightarrow \mathscr{A}$, i.e. $F(g) \in \mathscr{A} \forall g$ such that $F(\mathrm{~g})$ is a measurable function on $G$ and $\|F\|:=\int\|F(g)\| d g<\infty$. $\mathscr{A}_{1}^{G}$ is a vectorspace in a natural manner and in particular a Banachspace. Given two elements $F, G \in \mathscr{A}_{1}^{G}$ there exists a product defined by $(F * G)(g)=\int F(h) \alpha_{h} G\left(h g^{-1}\right) d h$ which fulfills the property $\|F * G\|$ $\leqq\|F\|\|G\|^{1}$. Furthermore there exists on $\mathscr{A}_{1}^{G}$ an involution defined by $\left(F^{*}\right)(g)=\alpha_{g}\left(F\left(g^{-1}\right)\right)^{*} \Delta\left(g^{-1}\right)$ with the properties $F^{* *}=F$ and $\left\|F^{*}\right\|=\|F\|$. The product and the involution have all properties which are required for converting $\mathscr{A}_{1}^{G}$ into a $B^{*}$-algebra. Having established that $\mathscr{A}_{1}^{G}$ is a $B^{*}$-algebra one defines a homomorphism of $\mathscr{A}$ resp. $G$ into the bounded linear operators acting on $\mathscr{A}_{1}^{G}$ by $(H(g) F)(h)=\alpha_{g} F\left(g h^{-1}\right), g \in G$ and $(H(x) F)(g)=x F(g), x \in \mathscr{A}$. The norm of these operators are given by $\|H(g)\|=1$ and $\|H(x)\|=\|x\|$. The main result of Doplicher, Kastler, and Robinson is the following:

[7] Theorem 3. There is one-to-one correspondence between covariant representations $\pi$ of $\mathscr{A}$ and representations $\hat{\pi}$ of $\mathscr{A}_{1}^{G} \cdot \pi \rightarrow \hat{\pi}$ is given by $\hat{\pi}(F)=\int \pi\left(F(g) \varrho(g) d g, F \in \mathscr{A}_{1}^{G}\right.$ and $\varrho(g)$ the given strongly continuous unitary representation of $G$ implementing the automorphisms $\alpha_{g}$. The integral is defined in the strong operator-topology. The inverse correspondence is defined by the relations $\pi(x) \hat{\pi}(F)=\hat{\pi}(H(x) F), x \in \mathscr{A}$ and $\varrho(g) \hat{\pi}(F)=\hat{\pi}(H(g) F), g \in G$.

\footnotetext{
${ }^{1} d g$ denotes a left invariant Haar measure and $\Delta$ the modul function.
} 
By means of this correspondence between representations of $\mathscr{A}_{1}^{G}$ and covariant representations of $\mathscr{A}_{1}$ we have a natural map $\phi$ from the states of $\mathscr{A}_{1}^{G}$ into the states of $\mathscr{A}$. Since for a given covariant representation might exist several different group representations implementing the automorphisms $\alpha_{g}$ the map $\phi$ will in general be many to one. But we have

III.2. Theorem. The image of the mapping $\phi$ from $E\left(\mathscr{A}_{1}^{G}\right)$ into $E(\mathscr{A})$ is onto $E_{c}(\mathscr{A})$, i.e. $\phi E\left(\mathscr{A}_{1}^{G}\right)=E_{c}(\mathscr{A})$.

Proof. From Lemma II.2 follows $\phi E\left(\mathscr{A}_{1}^{G}\right) \subset E_{c}(\mathscr{A})$. It remains to show the converse inclusion. Let $J \in \mathscr{A}_{1}^{G}$ such that $J(g)$ is a continuous function with compact support. $\left(J^{*} * F * J\right)(g)$ is a continuous function in $g$ ([7], Theorem 2). In particular $F \rightarrow\left(J^{*} * F * J\right)(1)$ defines a linear positive map $\Psi_{J}$ from $\mathscr{A}_{1}^{G}$ into $\mathscr{A}$. Hence the transposed map $\Psi_{J}^{\prime}$ sends positive linear forms of $\mathscr{A}$ into positive linear forms of $\mathscr{A}_{1}^{G}$. This map is given by $\left.\Psi_{J}^{\prime} \omega(F)=\omega\left(J^{*} * F * J\right)(1)\right)$. ([7], Lemma 5). The rest of the proof of Theorem III.2 will be given in the following two lemmas.

III.3. Lemma.Let $\omega \in E_{c}(\mathscr{A})$ then exists a sequence $J_{n} \in \mathscr{A}_{1}^{G}$ continuous and with compact support such that $\phi \circ \Psi_{J_{n}}^{\prime} \omega$ converge in norm to $\omega$.

Proof. We have

$$
\left(J^{*} * F * J\right)(1)=\int \alpha_{g}\left(J^{*}\left(g^{-1}\right)\right) \alpha_{g}\left(F\left(g h^{-1}\right)\right) \alpha_{h}\left(J\left(h^{-1}\right)\right) \Delta\left(g^{-1}\right) d g d h .
$$

From this follows

$$
\Psi_{J}^{\prime} \omega(F)=\int d g d h \omega\left(\alpha_{g}\left(J^{*}\left(g^{-1}\right)\right) \alpha_{g}\left(F\left(g^{-1} h\right)\right) \alpha_{h}\left(J\left(h^{-1}\right)\right)\right) \Delta\left(g^{-1}\right) .
$$

Now construct $\phi \circ \Psi_{J}^{\prime} \omega(x)$ by replacing $F(g)$ by $x \cdot \delta(g)$ where denotes the Dirac measure at the identity of $G$. Hence we get

$$
\phi \circ \Psi_{J}^{\prime} \omega(x)=\int d g \omega\left(\alpha_{g} J^{*}\left(g^{-1}\right) x J\left(g^{-1}\right)\right) \Delta\left(g^{-1}\right) .
$$

Since we have assumed that $1 \in \mathscr{A}$ we can put $J_{n}(g)=1 f(g), f(g)$ continuous function with compact support on $G$. Thus we get

$$
\phi \circ \Psi_{J_{n}}^{\prime} \omega(x)=\int d g\left|f\left(g^{-1}\right)\right|^{2} \alpha_{g}^{\prime} \omega(x) \Delta\left(g^{-1}\right) .
$$

Since $\alpha_{g}^{\prime}$ acts strongly continuous we can choose $f_{n}(g)$ such that $\left|f_{n}\left(g^{-1}\right)\right|^{2} \Delta\left(g^{-1}\right)$ converges to $\delta(g)$ in the dual space of the continuous on $G$. Hence $\phi \circ \Psi_{J_{n}}^{\prime} \omega$ converges in norm to $\omega$.

This lemma tells us that $\phi E\left(\mathscr{A}_{1}^{G}\right)$ is normdense in $E_{c}(\mathscr{A})$. Therefore the proof of Theorem III.2 is established by the following

III.4. Lemma. The image of $E\left(\mathscr{A}_{1}^{G}\right)$ under the map $\phi$ is closed in norm.

Proof. Let $\omega$ be a limit point of $\phi E\left(\mathscr{A}_{1}^{G}\right)$ then exists a sequence $\omega_{n} \in \phi E\left(\mathscr{A}_{1}^{G}\right)$ such that $\left\|\omega_{n}-\omega\right\| \rightarrow 0$. 
Let $\hat{\omega}_{n} \in E\left(\mathscr{A}_{1}^{(i)}\right)$ such that $\phi \hat{\omega}_{n}=\omega_{n}$. Denote by $\hat{\pi}=\sum_{n} \oplus \hat{\pi}_{\hat{\omega}_{n}}$ the representation of $\mathscr{A}_{1}^{G}$ induced by $\left\{\omega_{n}\right\}$. By the correspondence-theorem exists a covariant representation $\pi$ of $\mathscr{A}$ such that $\phi \hat{\omega}_{\xi}=\omega_{\xi}$ for every $\xi \in \mathscr{H}_{\hat{\pi}}$. Since the vectorstates are norm closed (R. V. Kadison [12]) exist a vector $\eta \in \mathscr{H}_{\hat{\pi}}$ with $\omega=\omega_{\eta}=\phi \hat{\omega}_{\eta}$.

After the proof of Theorem III.2 we can complete the proof of Theorem III.1. Let $\pi$ be a representation of $\mathscr{A}$ with $\pi \eta E_{c}$ then it is the direct sum of cyclic representation. $\pi=\sum_{i \in I} \oplus \pi_{\omega_{i}}$. Since $\omega_{i}$ belongs to $E_{c}$ exists $\hat{\omega}_{i} \in E\left(\mathscr{A}_{1}^{G}\right)$ such that $\phi \hat{\omega}_{i}=\omega_{i}$. Now $\sum_{i \in I} \oplus \hat{\pi}_{\hat{\omega}_{i}}$ is a representation $\hat{\pi}$ of $\mathscr{A}_{1}^{G}$. Let $\pi_{1}$ be corresponding covariant representation of $\mathscr{A}$ then one checks easily that $\pi$ is a subrepresentation of $\pi_{1}$. This proves Theorem III.1.

After having established necessary and sufficient conditions for the existence of covariant extension of a given representation we will ask next for the class of quasicovariant representations.

Before we are going into the details of the discussions we have to recall the condition for quasi equivalence in terms of states. Let $\pi_{1}$ and $\pi_{2}$ be two quasi equivalent representations then every normal state of $\pi_{1}$ defines a normal state on $\pi_{2}$ and vice versa. Hence $\pi_{1}$ and $\pi_{2}$ have the same sets of normal states. Now the set of normal states of a representation $\pi$ is normclosed and coincides with the convex closure of its vectorstates.

III.5. Definition. Let $\pi$ be a representation then we denote

1. $E(\pi)$ the set of all vectorstate of $\pi$.

2. $\overline{C_{0}} E(\pi)$ the norm closed convex hull of $E(\pi)$.

Since for any state $\omega \in \overline{C_{0}} E(\pi)$ the representation $\pi_{\omega}$ is quasi equivalent to a subrepresentation of $\pi$ follows that $\sum_{\omega \in \overline{C_{0}} E(\pi)} \oplus \pi_{\omega}$ is quasi equivalent to $\pi$. But this implies two representations $\pi_{1}$ and $\pi_{2}$ are quasi equivalent if $\overline{C_{0}} E\left(\pi_{1}\right)=\overline{C_{0}} E\left(\pi_{2}\right)$.

After this discussion of quasi equivalent representations we can state the result.

III.6. Theorem. Let $\pi$ be a representation of $\mathscr{A}$ then $\pi$ is quasi covariant if and only if

ג) $\pi \eta E_{c}$ and

B) $\alpha_{g}^{\prime} \overline{C_{0}} E(\pi)=\overline{C_{0}} E(\pi)$ for all $g \in G$.

Proof. Let $\pi$ be a quasi covariant representation then exists a covariant representation $\pi_{1}$ which is quasi equivalent to $\pi$. Hence $E(\pi) \subset E_{c}$ and since $E_{c}$ is convex and norm closed follows $E_{c} \supset \overline{C_{0}} E\left(\pi_{1}\right)=\overline{C_{0}} E(\pi)$ but this implies $\pi \eta E_{c}$. Since $\pi_{1}$ is a covariant representation follows 
$\alpha_{g}^{\prime} E\left(\pi_{1}\right)=E\left(\pi_{1}\right)$ and hence also the convex closure of $E(\pi)$ is invariant under the action of $\alpha_{g}^{\prime}$.

Let now $\pi$ fulfil the conditions $\alpha$ ) and $\beta$ ) then by Theorem III.1 exists a covariant representation $\pi_{1}$ such that $\pi$ is a subrepresentation of $\pi_{1}$. Let now $P$ be the central carrier of $\pi$ (in the center of the weak closure of $\pi_{1}$ ) then $P \pi_{1}$ is quasi equivalent to $\pi$. It remains to show that $P \pi_{1}$ is a covariant representation. To this end let us denote by $\varrho(g)$ the strongly continuous unitary representation of $G$ on $\mathscr{H}_{\pi_{1}}$ implementing the automorphisms. Let $\xi \in \mathscr{H}_{\pi_{1}}$ such that $P \xi=\xi$ then by condition $\beta$ $\varrho(g) \xi$ defines a normal state of $P \pi_{1}$ and since the representations $P \pi_{1}$ and $(1-P) \pi_{1}$ are disjoint follows $P \varrho(g) \xi=\varrho(g) \xi$. This implies $P$ commutes with $\varrho(g)$ and hence $P \pi_{1}$ is a covariant representation. This proves the theorem.

Remarks. 1. We refrain from investing conditions under which a representation is covariant. The necessary condition that $E(\pi)$ is invariant under $\alpha_{g}^{\prime}$ is not sufficient for solving the problem as we will see in an example. As far as I have looked into this problem the invariance of $E(\pi)$ will probably be sufficient in the cases where $\pi^{\prime \prime}$ is purely infinite and in the case where $\pi^{\prime \prime}$ is finite only when the coupling is smaller or equal to one, this means if $\pi^{\prime}$ is small compared to $\pi^{\prime \prime}$.

Example. Let $\mathscr{A}$ be the continuous functions on the real line $\mathbb{R}$ vanishing at infinity with the translations as automorphism groups. Let $\pi_{0}$ be the natural representation in $\mathscr{L}_{1}(\mathbb{R})$ and $P$ be a projection in $\pi_{0}^{\prime \prime}$ such that $P \neq 0$ and $1-P \neq 0$. Let $\mathscr{K}$ be any Hilbertspace of at least two dimensions. Define $\mathscr{H}=P \mathscr{L}_{2}(\mathbb{R}) \otimes \mathscr{K} \oplus(1-P) \mathscr{L}_{2}(\mathbb{R})$ and $\pi(x)=P \pi_{0}(x) \otimes 1 \oplus(1-P) \pi_{0}(x)$. One sees immediately $E(\pi)=E\left(\pi_{0}\right)$ and hence $E(\pi)$ is invariant under the action of $\alpha_{g}^{\prime}$. However $\alpha_{g}$ is not unitary implementable since multiplicity is an unitary invariant.

2. From the way we have constructed states on the covariancealgebra $\mathscr{A}_{1}^{G}$ in the proof of Theorem III.2 follows that there exists a faithful covariant representation of $\mathscr{A}$ (compare also [7], Lemma 5). From this we will see that a twosided ideal in $\mathscr{A}$ is kernel of a covariant representation if and only if it is invariant.

Acknowledgement. Parts of this work has been done during a visit at the University of Aix-Marseille. I would like to thank D. Kastler for his warm hospitality.

\section{References}

1. Haag, R., and D. Kastler: An algebraic approach to quantum field theory. J. Math. Phys. 5, 834 (1964).

2. Dixmier, J.: Les $C^{*}$-algèbres et leurs représentations. Paris: Gauthier-Villars 1964.

3. Kadison, R. V., and J. Ringrose: Derivations and automorphisms of operator algebras. Commun. Math. Phys. 4, 32 (1967). 
4. Sakai, S.: Derivations of $W^{*}$-algebras. Ann. Math. 83, 273 (1966).

5. Kadison, R. V.: The energy momentum spectrum of quantum fields. Commun. Math. Phys. 4, 258 (1967).

6. Doplicher, S., R. V. Kadison, D. Kastler, and D. W. Robinson: Asymptotically abelian systems. Commun. Math. Phys. 6, 101 (1967).

7. - D. Kastler, and D. W. Robinson: Covariance algebras in field theory and statistical mechanics. Commun. Math. Phys. 3, 1 (1966).

8. Dell'Antonio, G. F.: On some groups of automorphisms of physical observables. Commun. Math. Phys. 2, 384 (1966).

9. Borchers, H. J.: On groups of automorphisms with semi-bounded spectrum. Preprint.

10. - Energy and momentum as observables in quantum field theory. Commun. Math. Phys. 2, 49 (1966).

11. Dixmier, J.: Les algèbres d'opérateurs dans l'espace Hilbertien. Paris: GauthierVillars 1957.

12. Kadison, R. V.: States and representations. Trans. Am. Math. Soc. 103, 304 (1962).

H. J. Borchers

Institut für Theoretische Physik

der Universität

3400 Göttingen 